\title{
USING INDIVIDUAL-BASED MODELING TO BETTER UNDERSTAND THE HIDDEN CURRICULUM OF ENGINEERING
}

\author{
Robyn Paul, Laleh Behjat, and Robert Brennan \\ University of Calgary \\ rmpaul@ucalgary.ca
}

\begin{abstract}
Engineering culture perpetuates norms that are unwelcoming to minoritized identities, particularly women and racialized folks. A theory useful for understanding this is "hidden curriculum" which describes the assumptions and beliefs that are unintentionally and implicitly taught in engineering education. This paper outlines an initial conceptual model for using IBM (individual-based modeling) to better understand the hidden curriculum of engineering. We provide an overview of the driving question behind the model design, the agents and their attributes, the rules and processes which change these attributes, and the scale of the model. This overview of the model building process provides insight into the model design for simulating and better understanding the perpetuation of the hidden curriculum within engineering education.
\end{abstract}

Keywords: Hidden curriculum : Individual based modeling : Agent based modeling : Model Development

\section{INTRODUCTION}

Women have always been an underrepresented group in engineering, however percentages of women entering engineering in the past 35 years has remained stagnant at about 20\% [15]. Much of the outreach and gender research within engineering has been shown to "universalize white, western, middle class women" [2]. This approach continues to perpetuate the underlying cultural ideals of engineering, and maintains the male-dominated and hierarchical classroom environments with a presumed neutrality and meritocracy [4].

A theory that is helpful in understanding the underlying cultural norms of education systems is hidden curriculum, which is the idea that "particular assumptions, beliefs, values, or attitudes manifest themselves implicitly and inadvertently in schooling, learning, and professional environments" [19]. Over time, these hidden lessons become a normalized part of the culture that continue to perpetuate ideologies which often privilege certain forms of knowledge and ways of being, while oppressing others.

Individual-based models (IBM) are being increasingly used to study and explore social and educational phenomena. IBMs use computer simulations to study system-level phenomena that emerge from adaptive behaviour of individual agents. IBM simulations represent complex systems, where through simple sets of rules they can model real-world phenomena.

Previously, we have described our approach for exploring the use of IBMs within engineering to provide an experimental method for easily testing research questions and interventions without physically using real participants and classroom environments [3, 13]. Although IBMs are not a replacement for these, they provide a first step at understanding how individuals and the system might react to changes, which can inform experimental design.

This paper outlines an initial conceptual model for using IBM to better understand the hidden curriculum of engineering. We provide an overview of the driving question behind the model design, the agents and their attributes, the rules and processes which change these attributes, and the scale of the model. This overview of the model building process provides insight into the model design for simulating and better understanding the perpetuation of the hidden curriculum within engineering education.

\section{LITERATURE BACKGROUND}

\subsection{Hidden Curriculum in Engineering}

As our education systems have been formalized over the past centuries, scholars have studied the underlying beliefs and values that are being taught in educational settings. Hidden curriculum is the theory that "particular assumptions, beliefs, values, or attitudes manifest themselves implicitly and inadvertently in schooling, learning, and professional environments" [19]. The theory of hidden curriculum has been studied since the middle of the twentieth century [10], with some early scholars describing it simply as "what schooling does to people" [18]. Students are not just learning the content being taught, but there are also the hidden lessons in our classrooms. For example, within K-12 schools, students are taught very early that they will be rewarded for conforming to institutional expectations, such as patiently waiting, listening quietly, neatness, and punctuality. These lessons are often not part of the explicit educational goals, however they are requirements for successful progression through schooling [11]. Students who do not comply with these 
unwritten rules will be disadvantaged relative to their peers.

Within engineering specifically, Idalis Villanueva and her team have done significant work at looking at the hidden curriculum of engineering. For example, they explored engineering faculty members awareness of professionalism as a hidden curriculum [19] and they have developed a mixed-methods survey to explore hidden curricula in engineering [20]. In one of their publications, they provide six examples of hidden curriculum statements to research participants, for example, the ultimate goal of an engineering degree is to get a well-paying job; not everyone can be an engineer; and engineering education is harder, more time consuming, and expensive because it has a direct impact on safety [5].

It is important to recognize that these hidden lessons often become part of the unconscious mind and a normalized part of our culture. These invisible and normalized hidden curricula bring light to the ideologies being perpetuated, usually privileging some and oppressing other forms of knowledge and ways of being. Foundational to these ideologies, is the importance of maintaining a reputation of neutrality, that the education system in itself is and does not take any political stance. Michael Apple argues strongly against this, because not only do schools act as "powerful agents in the economic and cultural reproduction of class relations," but schools play a significant role in fostering a specific cultural capital $[1$, p. 25]. Although education may appear neutral on the surface, choices are made in the types of knowledges that we teach, and these choices have underlying ideological assumptions on our social and economic values and culture. Hidden curricula thus is typically promoted by those who are in a privileged position, where it benefits the powerful and oppresses the marginalized [11].

\subsection{Individual-Based Modeling}

As our world becomes increasingly complex, "simple models no longer suffice to answer many of our questions" [21]. However, with the progression of computer modeling and processing power, several methodologies are able to support the analysis of complex systems that are beyond the capability of our human brains. Individual-based models (IBMs) are representations of individual organisms and agents. IBMs use simulations to study system-level phenomena that emerge from adaptive behaviour of individual agents $[6,7]$.

Individual-based modeling was chosen as a tool for this research because IBMs model individual agents in a heterogenous population, comparatively to other modeling techniques which use mathematical equations that can only determine homogeneous population averages. For my research, which uses frameworks from feminism and queer theory, this heterogeneous approach to modeling is important. Although it is difficult to make conclusions based on individual agents, within an IBM "it is possible to examine the history and life of any one individual in the model" [21, p.33]. IBM will therefore allow me to look at different subsets of individuals and how changes impact specific demographics, rather than only having data about the population-level changes.

\section{IBM in Social Science and Education}

Early IBM techniques were used to model networked systems such as ant foraging, bird flocking behaviour, traffic flow, and crowd behaviours. Recently, these techniques have been applied to model a wider variety of complex social systems and educational environments. $\mathrm{Gu}$ and Blackmore [8] conducted a systematic review of IBM applications to higher education. They found IBMs have modeled "the university system, university collaboration, academic activities, application and enrolment, and student performance" (p. 894). To highlight a recent example, Seman et al. [16] describe their approach to using IBM to model project-based learning in electrical engineering contexts. In their PBL simulation, they found students' incoming knowledge was very important for them to be able to participate fully on the teams. To mitigate this, they found creating PBL teams with the greatest diversity of students increased the sharing of experiences and knowledge, leading to stronger team dynamics and project outcomes [16]. Within our lab, we are using IBM to model cheating behaviours in engineering classrooms, where we were able to better understand the impact of harder assignments, a cheating culture, and poor prerequisites on engineering students' cheating behaviours [3, 13].

IBM has also been used to model marginalized populations, and support discourse on complex sociopolitical issues. Paré et al. [12] found that macro-level structural phenomena such as marginalization and resilience emerged from individual-level behaviour, supporting better understanding of queer gender and sexual expression. These types of models are not only powerful for understanding emergent behaviours, but they can be used to engage in conversations about the topics. For example, using an IBM as a tool, participants engaged in conversations about complex sociopolitical issues such as ethnocentrism and racial segregation [9]. Within these models there is an essential component of designing activities which support critical discourse that help to contextualize the computer algorithms and visualizations. For example, while engaging in the IBM on queer gender and sexual expression, the authors integrated first-person narratives of gender and sexuality-based marginalization and resilience into the simulation activity [12]. Using IBM as a discussion tool not only provides space for contextualizing technology and engineering design, but the models also deepen the technical inquiry and quality of technical knowledge [17]. 


\section{CONCEPTUAl Model DeSign}

The model design is in the early states, and we will describe the first few steps of the modeling cycle based on Grimm and Railsback's [7] approach. They outline the six tasks of the modeling cycle as show in Fig. 1 below. The process of modeling is iterative, and it is important to continuously cycle and repeat the tasks.

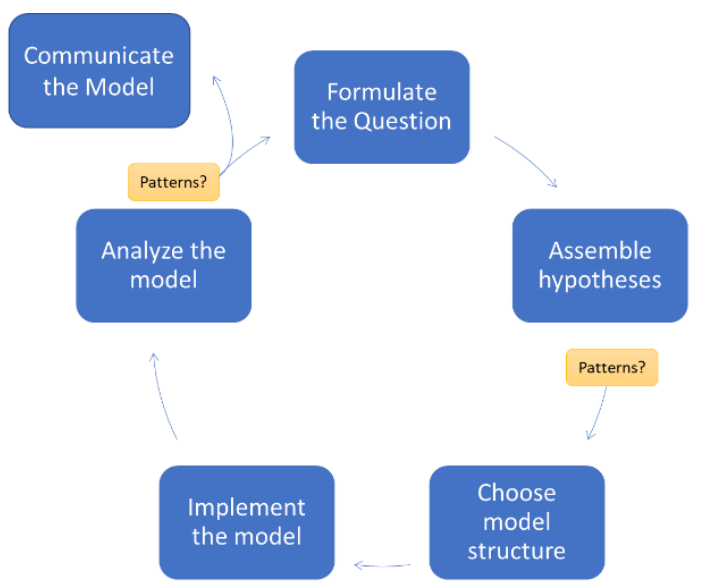

Fig. 1. Grimm and Railsback [7] modeling cycle.

\subsection{Formulate the Question}

The first task is to formulate the research question. Depending on the research, questions can be clearly articulated from the beginning, or they can frame a general desire to understand an area without a clear question [21]. Throughout the iterative modeling cycle, the research question is continuously refined and defined.

It is important when developing the research question to ensure that the area of research is a good fit for an individual-based modeling approach. Specifically, IBM tends to be more appropriate for heterogenous groups, environments with randomness, variables which are discrete, dynamic systems, and where the individual level behaviour is known (not the aggregate). Table 1 below summarizes how research into the hidden curriculum of engineering is well suited for IBM.

This work will be doing exploratory modeling, where we aim to explore the patterns of engineering culture which emerge from model development (contrary to phenomenabased modeling which aims to create a model that represents a reference pattern) [21]. For that reason, we are using a bottom-up design, where there is a general understanding of the phenomena without necessarily having a clear, well-defined research question. The conceptual model is designed from the bottom-up, with the research question being developed through the iterative development process [21].
Table 1. How IBM is a good fit for exploring this work.

\begin{tabular}{|c|c|}
\hline IBM guidelines & Fit with this work \\
\hline $\begin{array}{l}\text { Heterogeneous } \\
\text { (not } \\
\text { homogeneous) }\end{array}$ & $\begin{array}{l}\text { In social environments, the } \\
\text { heterogeneity of individuals affects } \\
\text { the overall patterns we observe in } \\
\text { society. Additionally, modeling } \\
\text { heterogeneity allows for greater } \\
\text { complexity of individuals' } \\
\text { interactions. }\end{array}$ \\
\hline $\begin{array}{l}\text { Random } \\
\text { (not determined) }\end{array}$ & $\begin{array}{l}\text { Individuals make decisions based } \\
\text { on their environment and an } \\
\text { element of randomness. Their } \\
\text { decisions are not determined. }\end{array}$ \\
\hline $\begin{array}{l}\text { Discrete } \\
\text { (not continuous) }\end{array}$ & $\begin{array}{l}\text { Each "agent" in the model } \\
\text { represents an individual. You } \\
\text { cannot have } 0.1 \text { of an agent. }\end{array}$ \\
\hline $\begin{array}{l}\text { Dynamic } \\
\text { (not static) }\end{array}$ & $\begin{array}{l}\text { Individuals take actions in the } \\
\text { model with the passing of time. } \\
\text { This dynamic feature moves } \\
\text { beyond a static snapshot of time, } \\
\text { and allows for a dynamic } \\
\text { understanding. }\end{array}$ \\
\hline $\begin{array}{l}\text { Individual } \\
\text { behaviour } \\
\text { known } \\
\text { (not aggregate) }\end{array}$ & $\begin{array}{l}\text { Often it is easier to consider the } \\
\text { individual actions, as we have a } \\
\text { harder time conceptualizing the } \\
\text { resulting aggregate patterns } \\
\text { (particularly when observing } \\
\text { societal biases). }\end{array}$ \\
\hline
\end{tabular}

Currently the phenomena being studied can be summarized by two exploratory questions: (1) What is the impact of hidden curriculum on engineering students' learning and approach to engineering work? (2) How does hidden curriculum specifically impact minoritized students?

\subsection{Assemble the Hypothesis}

To build a model, we must have a hypothesis which we are testing. These hypotheses are based on both theory and experience [7]. The theory provides a framework, and empirical knowledge of those who know the system well allows for refinement of this framework.

There are two primary current working hypotheses of this model. Firstly, we theorize that when engineering content is taught embedded with hidden curriculum messages, students take these messages into the way in which they approach engineering. This is based on the theory of hidden curriculum, whereby it is an understanding that students are learning are learning more than just the content being taught.

Secondly, we posit that students with minoritized identities will be more likely to feel tension with engineering when content is taught embedded with hidden curriculum messages.

Both these hypotheses also come from personal experience. Through conversations and workshops with 
many individuals across Canada on hidden curriculum, these phenomena are becoming more and more clear based on the lived experiences that others have shared with me. For example, after a recent workshop with high school physics teachers, a participant reached out to me to thank me for giving her words to label the tension she was feeling when she partook in physics workshops with other teachers, which are often very masculinized and hierarchical spaces. This tension that people with minoritized identities feel can lead to discouragement and isolation.

\subsection{Choose Model Structure}

The next step is to translate the hypothesis into a model structure. Typically this includes selecting the necessary agents, defining the attributes that cause changes to these agents, and defining the processes and rules which "quantify when, how much, and how fast the variables change" [7, p.29].

Throughout this process, it is important to keep in mind the most important IBM design principle: START SIMPLE (Wilensky). Although when modeling we often want to consider all characteristics of an agent (for example, age, ethnicity, gender, preferences of a student), we need to consider only the essential characteristics relevant for our research question. This will expand and change as the model develops, but the simpler we are able to start the model, the easier we can test and iteratively develop the design.

We also need to consider both spatial scale and temporal scale, which each have two aspects: grain (smallest slice to consider) and extent (total amount covered by model).

3.3.1. Model Agents and Attributes. The first iteration of the model will include two main agents: students and teachers.

The student agent, will be defined by four attributes:

- technical-knowledge

- HC-knowledge

- HC-awareness

- $\quad$ eng-identity

The technical-knowledge attribute represents how much a student has progressed through their academic career and is gaining the necessary technical knowledge for engineering

The HC-knowledge attribute represents how much a student has been exposed to the hidden curriculum of engineering and has adopted these perceptions and assumptions within their framing of engineering.

The HC-awareness attribute represents a students' awareness of the $\mathrm{HC}$ that exists within engineering and their ability to critically consider and take into account the HC when making engineering decisions.

And lastly, the eng-identity attribute is the extent to which the student feels they fit or belong in engineering.

The teacher agent will be defined by three attributes:
- technical-teaching

- HC-teaching

- HC-critical

The technical-teaching attribute represents how well the teacher is able to convey technical engineering content to the students. The HC-teaching attribute represents how much the teacher reinforces and perpetuates the $\mathrm{HC}$ of engineering in their teaching. And the HC-critical represents the teachers' awareness and critique of the $\mathrm{HC}$ in their teaching approach.

For example, a teacher-agent which has a high $\mathrm{HC}$ teaching and a high HC-critical, it would mean that they continue to perpetuate many of the hidden curricula of engineering, however they also critique and deconstruct these $\mathrm{HC}$ whenever possible. Perhaps they run their course in a very hierarchical manner and talk about engineering as an elitist degree, but they also give equal weight to the social element of engineering within their technical problem-solving examples.

3.3.2. Model Processes and Rules. The initial model will have some very simple processes and rules to test the functionality of the basic framework that will be built on with time. The processes or rules can be considered as the agent behaviours. The main process or agent interaction is that the teacher will "teach" the students. Each of the student agent attributes will increase based on the teacher agent attributes. This increase will be random for each student, which represents the different levels of learning within a classroom.

The students' eng-identity attribute will provide a secondary set of processes and rules. A high level of engidentity represents students who feel they "fit' or belong within engineering, and a low level represents students who feel isolated and disconnected with engineering. As their HC-knowledge increases, if they have a low eng-identity it will be lowered, and if they have a high eng-identity it will be increased. The assumption within this process is that students who feel more connected to engineering will feel more connected to the hidden curriculum of engineering (for example, they may feel proud of the masculinized and elitist culture of engineering). This assumption will be refined and adapted with future model iterations.

The HC-awareness will also impact the interaction of the eng-identity attribute. As a student develops awareness of the hidden curriculum of engineering (i.e. their $\mathrm{HC}$ awareness increases), they are more easily able to adapt to the impact of HC-knowledge on their eng-identity. This is based on research which shows that teaching minoritized students about the hidden curriculum of engineering increases their persistence in engineering [5].

3.3.3. Model Scale. The conceptual model currently relies on relationship for model processes, thus spatial scale of the model considers the relationships. The grain of the relationships includes one-on-one interactions between the teacher and the student in a classroom environment. At 
this time, there is no consideration of peer-to-peer interactions or mentorship relationships, although it is expected that future model iterations might consider these.

The extent of the spatial scale will consider one medium sized University engineering program with about 1000 first-year students.

The grain of the temporal scale (i.e. time step in the model) will be on a weekly basis. The reason for this grain is that typically students receive feedback on their knowledge gains approximately weekly, and this feedback provides them with information on their knowledge acquisition and progress in their degree.

The extent of the temporal scale will investigate the data throughout a full University degree (i.e. 8 semesters with about 13 weeks each). For the data analysis process, we may consider the agents' attributes after each semester and/or year students are exposed to different sets of teachers.

\section{CONCLUSIONS AND NeXT STEPS}

This paper provided an initial conceptual overview of using individual-based modeling to model the hidden curriculum of engineering. A simple initial model was conceptualized that showed the basic framework of hidden curriculum theories being applied to the IBM environment.

Future work will create and test a preliminary model, and aim to expand and refine this model to test the impact of interventions in the classroom.

\section{References}

[1] Apple, M. (2013). Ideology and curriculum. Routledge.

[2] Beddoes, K. (2011). Engineering education discourses on underrepresentation: Why problematization matters. International Journal of Engineering Education, 27(5), $1117-1129$

[3] Brennan, R., Hermanson, G., Nelson, N., Paul, R., \& Sullivan, M. (2019). Using agent-based modelling for preliminary EER experimental design. Canadian Engineering Education Association (CEEA).

[4] Cech, E. A. (2013). The (mis) framing of social justice: Why ideologies of depoliticization and meritocracy hinder engineers' ability to think about social injustices. In Engineering education for social justice (pp. 67-84). Springer, Dordrecht.

[5] Gelles, L. A., Youmans, K. L., Villanueva-Perez, C., \& Di Stefano, M. (2019). Hidden Curriculum Advocacy and Resources for Graduate Students in Engineering. CoNECD Conference, 1-16.

[6] Grimm, V., Berger, U., Bastiansen, F., Eliassen, S., Ginot, V., Giske, J., Goss-Custard, J., Grand, T., Heinz, S. K., Huse, G., Huth, A., Jepsen, J. U., Jørgensen, C., Mooij, W.
M., Müller, B., Pe'er, G., Piou, C., Railsback, S. F., Robbins, A. M., ... DeAngelis, D. L. (2006). A standard protocol for describing individual-based and agent-based models. Ecological Modelling, 198(1-2), 115-126.

[7] Grimm, V., \& Railsback, S. F. (2005). Individual-based modeling and ecology (Vol. 8). Princeton university press.

[8] Gu, X., \& Blackmore, K. L. (2015). A systematic review of agent-based modelling and simulation applications in the higher education domain. Higher Education Research and Development, 34(5), 883-898.

[9] Hostetler, A., Sengupta, P., \& Hollett, T. (2018). Unsilencing Critical Conversations in Social-Studies Teacher Education using Agent-based Modeling. Cognition and Instruction, 36(2), 139-170.

[10] Kentli, F. D. (2009). Comparison of hidden curriculum theories. European Journal of Educational Studies, 1(1968), 83-88.

[11] Margolis, E. (2001). The hidden curriculum in higher education. Psychology Press.

[12] Paré, D., Shanahan, M., \& Sengupta, P. (2020). Queering Complexity Using Multi-Agent Simulations Queering Complexity Using Multi-Agent Simulations Theoretical Background: Synergies between Complexity and Queer Theories. April.

[13] Paul, R., Eaton, S. E., Laird, G., Nelson, N., \& Brennan, R. (2020). Using agent-based modelling for eer experimental design: preliminary validation based on student cheating behaviours. Canadian Engineering Education Association (CEEA).

[14] Railsback, S. F., \& Grimm, V. (2019). Agent-Based and Individual-Based Modeling: A Practical Introduction (Second Edi). Princeton University Press.

[15] Sax, L. J., Allison Kanny, M., Jacobs, J. A., Whang, H., Weintraub, D. S., \& Hroch, A. (2016). Understanding the Changing Dynamics of the Gender Gap in Undergraduate Engineering Majors: 1971-2011. In Research in Higher Education (Vol. 57, Issue 5). Springer Netherlands.

[16] Seman, L. O., Hausmann, R., \& Bezerra, E. A. (2018). Agent-Based Simulation of Learning Dissemination in a Project-Based Learning Context Considering the Human Aspects. IEEE Transactions on Education, 61(2), 101-108.

[17] Sengupta, P., Dickes, A., \& Farris, A. (2020). Voicing Code as Recontextualization and Transitional Othering (in press). In Voicing Code in STEM - A Dialogical Imagination (pp. 87-117). MIT Press.

[18] Vallance, E. (1973). Hiding the Hidden Curriculum: An Interpretation of the Language of Justification in Nineteenth-Century Educational Reform. Curriculum Theory Network, 4(1), 5.

[19] Villanueva, I., Carothers, T., Di Stefano, M., \& Khan, M. T. H. (2018). "There is never a break": The hidden curriculum of professionalization for engineering Faculty. Education Sciences, 8(4). 
Proceedings 2021 Canadian Engineering Education Association (CEEA-ACEG21) Conference

[20] Villanueva, I., Gelles, L. A., Di Stefano, M., \& Youmans, K. (2019). Developing a mixed-method survey to explore hidden curriculum in Engineering Education. Northern Rocky Mountain Educational Research Association, $1653140,1653140$.
[21] Wilensky, U., \& Rand, W. (2015). An introduction to agent-based modeling: modeling natural, social, and engineered complex systems with NetLogo. MIT Press. 